\title{
GDNF Reduces Drug-Induced Rotational Behavior after Medial Forebrain Bundle Transection by a Mechanism Not Involving Striatal Dopamine
}

\author{
Jack L. Tseng, ${ }^{1}$ E. Edward Baetge, ${ }^{2}$ Anne D. Zurn, ${ }^{1}$ and Patrick Aebischer ${ }^{1}$ \\ ${ }^{1}$ Gene Therapy Center and Division of Surgical Research, Centre Hospitalier Universitaire Vaudois, Lausanne University \\ Medical School, 1011 Lausanne, Switzerland, and ${ }^{2}$ CytoTherapeutics, Providence, Rhode Island 02906
}

Parkinson's disease (PD) is characterized by the progressive loss of the substantia nigra (SN) dopaminergic neurons projecting to the striatum. Neurotrophic factors may have the potential to prevent or slow down the degenerative process occurring in PD. To that end, we examined whether low amounts of glial cell line-derived neurotrophic factor (GDNF) continuously released from polymer-encapsulated genetically engineered cells are able to prevent the loss of tyrosine hydroxylase immunoreactivity (TH-IR) in SN neurons and ameliorate the amphetamineinduced rotational asymmetry in rats that have been subjected to a unilateral medial forebrain bundle (MFB) axotomy. Baby hamster kidney (BHK) cells transfected with the cDNA for GDNF were encapsulated in a polymer fiber and implanted unilaterally at a location lateral to the MFB and rostral to the SN. ELISA assays before implantation show that the capsules release $\sim 5 \mathrm{ng}$ of GDNF/capsule per day. One week later, the MFB was axotomized unilaterally ipsilateral to the capsule placement. Seven days later, the animals were tested for amphetamine-induced rotational asymmetry and killed. The striatum was excised and analyzed either for catecholamine content or $\mathrm{TH}-\mathrm{IR}$, while the SN was immunostained for the presence of TH-IR. GDNF did not prevent the loss of dopamine in the striatum. However, GDNF significantly rescued TH-IR neurons in the SN pars compacta. Furthermore, GDNF also significantly reduced the number of turns per minute ipsilateral to the lesion under the influence of amphetamine. Improvement of rotational behavior in the absence of dopaminergic striatal reinnervation may reflect neuronal plasticity in the $\mathrm{SN}$, as suggested by the dendritic sprouting observed in animals receiving GDNF. These results illustrate that the continuous release of low levels of GDNF close to the $\mathrm{SN}$ is capable of protecting the nigral dopaminergic neurons from an axotomy-induced lesion and significantly improving pharmacological rotational behavior by a mechanism other than dopaminergic striatal reinnervation.

Key words: glial cell line-derived neurotrophic factor; medial forebrain bundle axotomy; Parkinson's disease; tyrosine hydroxylase; substantia nigra; polymer encapsulation
Parkinson's disease (PD) is a neurodegenerative disorder characterized by the progressive loss of dopaminergic neurons in the substantia nigra (SN) pars compacta, resulting in resting tremor, rigidity, bradykinesia, and postural imbalance (Goetz et al., 1989). Symptomatic therapy with the dopamine precursor levodopa, although effective in the first few years of administration, leads over time to deleterious side effects such as hallucinations and sudden freezing episodes (Goetz et al., 1989). A neuroprotective treatment that would slow down, prevent, or even reverse the degeneration process is, therefore, needed. Neurotrophic factors are known to play important roles in the development of neural tissue. They also play a vital role in the protection of neurons against various insults. Several neurotrophic factors, such as brain-derived neurotrophic factor (BDNF), have shown promise in the rescue of dopaminergic neurons in vitro. However, their effectiveness in vivo has been, for the most part, somewhat less promising (Knüsel et al., 1992; Hagg and Varon, 1993; Lapchak et al., 1993). Glial cell line-derived neurotrophic factor (GDNF) was

Received July 18, 1996; revised Sept. 27, 1996; accepted Oct. 8, 1996.

This study was supported by grants from the Swiss National Science Foundation and the Swiss National Program on Disorders of the Nervous System. We thank Dana Hornfeld, Anne Menoud, Meriem Tekaya, and Laurence Winkel for their excellent technical support.

Correspondence should be addressed to Dr. Patrick Aebischer, Gene Therapy Center and Division of Surgical Research, Centre Hospitalier Universitaire Vaudois, Pavillon 4, 1011 Lausanne, Switzerland.

Copyright (C) 1996 Society for Neuroscience $0270-6474 / 96 / 170325-09 \$ 05.00 / 0$ reported specifically to enhance the survival of midbrain dopaminergic neurons in vitro (Lin et al., 1993). GDNF also has been reported to exert protective effects on degenerating dopaminergic neurons in vivo. Injections of GDNF into the striatum of C57/B16 mice before 1-methyl-4-phenyl-1,2,3,6-tetrahydropyridine (MPTP) treatment prevent the decrease in dopamine levels (Tomac et al., 1995). A single intranigral injection of GDNF $24 \mathrm{hr}$ before a 6-OHDA nigral lesion rescues tyrosine hydroxylase immunoreactivity (TH-IR) for at least 2 weeks in rats (Kearns and Gash, 1995). Sauer et al. (1995) have reported that repeated intranigral injections of GDNF after a striatal 6-OHDA lesion also prevent the loss of nigral TH-IR in rats. A bolus injection of GDNF 4 weeks after a nigral 6-OHDA lesion in rats also maintains nigral dopamine levels (Hoffer et al., 1994) and TH-IR (Bowenkamp et al., 1995) for 5 weeks. More recently, Gash et al. (1996) have reported a bilateral improvement of the parkinsonian symptoms in primates unilaterally lesioned with the neurotoxin MPTP that have received three monthly bolus $100 \mu \mathrm{g}$ doses of GDNF into their lateral ventricles. A major concern with all of these reports, however, is the large amounts (tens to hundreds of micrograms) of GDNF being injected into the brain, because the actions of GDNF are limited not only to dopaminergic neurons. GDNF has been shown to increase choline acetyltransferase activity (ChAT) in cranial motor nuclei and spinal motoneurons in vitro and to prevent the death of motoneurons in vivo (Henderson et al., 1994; Zurn et al., 1994; Li et al., 1995; Yan et al., 1995; 
A

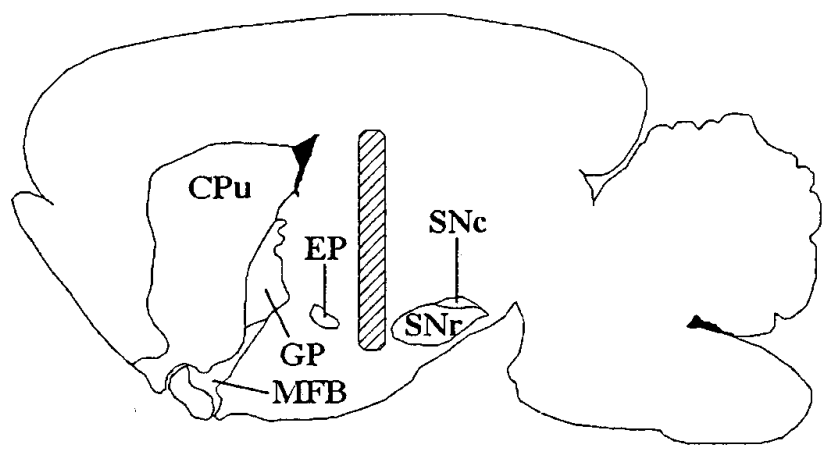

$B$

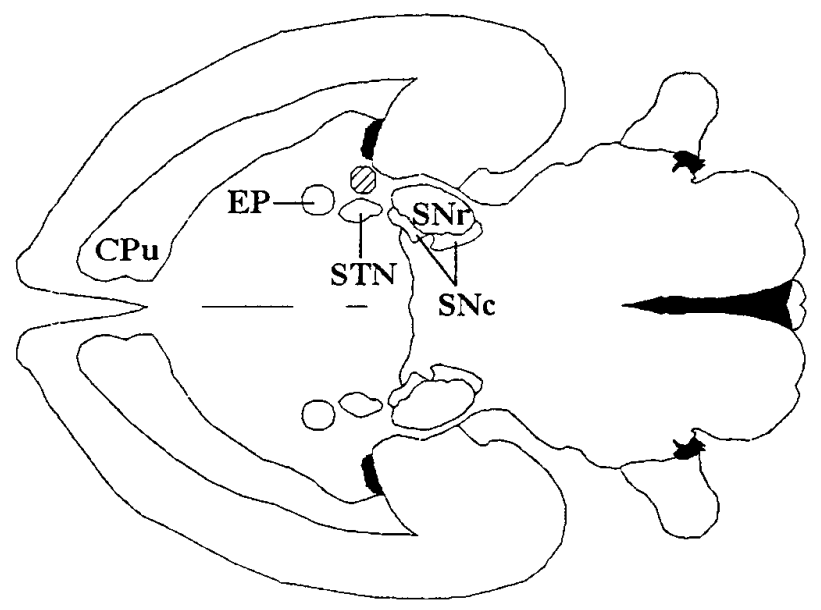

Figure 1. Diagram depicting the capsule implantation site in relation to the striatum, the substantia nigra, and the medial forebrain bundle. $A$, Sagittal view; $B$, horizontal view. The implanted capsule is represented by the hatched area. $C P u$, Caudate putamen; $G P$, globus pallidus; $E P$, enteropeduncular nucleus; $M F B$, medial forebrain bundle; $S N c$, substantia nigra pars compacta; $S N r$, substantia nigra pars reticulata; $S T N$, subthalamic nucleus [adapted from Paxinos and Watson (1986)].

Sagot et al., 1996). GDNF mRNA is expressed widely within the adult (N. A.-M. Pochon and P. Aebischer, unpublished observations) and embryonic rat brain (Schaar et al., 1993; Strömberg et al., 1993; Humpel et al., 1994; Springer et al., 1994). For these reasons, the ability to rescue neurons without the injection of massive amounts of factor would be advantageous for the clinical application of this factor.

One method of achieving continuous release of small amounts of GDNF relies on the transplantation of cells that have been engineered to release GDNF. Cell lines offer the advantages of unlimited availability, suitability for stable gene transfer via nonviral vectors, screening possibility for adventitious agents before transplantation, and establishment of certified cell banks. The risk of tumor formation can be controlled by the encapsulation technology with xenogeneic cell lines. Surrounding cells with a synthetic permselective membrane of appropriate molecular weight cutoff allows the inward diffusion of nutrients and the outward diffusion of neurotrophic factors; it also blunts that of immunocompetent molecules and excludes interaction with immunocompetent cells, therefore isolating the transplanted cells from the host immune system (Aebischer et al., 1991). In the case of capsule failure, the transplanted cells are rejected by the host immune system (Aebischer et al., 1991). This technique also allows the continuous delivery of neurotrophic factors within the nervous system while avoiding the potential problems associated with repeated invasive procedures. It recently has been applied in humans for the intrathecal delivery of recombinant human ciliary neurotrophic factor (CNTF) in amyotrophic lateral sclerosis patients (Aebischer et al., 1996). The present report shows that the continuous daily delivery of nanogram levels of GDNF by polymer-encapsulated engineered cells before medial forebrain bundle (MFB) axotomy can prevent in rats the degeneration of SN dopaminergic neurons. It also suggests the existence of an alternative mechanism for the control of pharmacologically induced rotational behavior in a lesioned nigrostriatal system.

\section{MATERIALS AND METHODS}

\section{Animals}

Adult female Wistar rats weighing 180-200 gm were obtained from IFFA-CREDO (L'Arbresle, France) and housed in a standard $12 \mathrm{hr}$ on/off light cycle with ad libitum access to food and water.

\section{Cells and cell encapsulation}

Baby hamster kidney (BHK) cells were transfected with a dihydrofolate reductase-based expression vector (pNUT, Baetge et al., 1986) containing the cDNA for rat GDNF (BHK-GDNF) via a calcium phosphate precipitation method (Zurn et al., 1994). The cDNA was synthesized by a reverse transcriptase PCR. The transfected cells were selected by increasing concentrations of methotrexate (Sigma, St. Louis, MO) over a period of 8 weeks. Nontransfected parent BHK cells were used as controls. All cells were cultured in DMEM containing $10 \%$ fetal calf serum (FCS), $1 \%$ penicillin/streptomycin, and $12 \mathrm{~mm}$ L-glutamine (all from Life Technologies, Paisley, Scotland). The cells were harvested with a standard dissociation medium (Sigma) and suspended in a 50/50 solution of culture medium and 3\% collagen (Zyplast, Collagen, Palo Alto, CA) at a density of $5 \times 10^{5} \mathrm{cells} / \mu \mathrm{l}$. Then the cell solution was injected into $500 \mu \mathrm{m}$ inner diameter poly(ether-sulfone) (PES) fibers (AKZO-Fiber Nobel AG, Wupperthal, Germany). The fibers were heat-sealed and cut at $5 \mathrm{~mm}$ intervals. The heat-sealed ends of the fibers were reinforced with a photopolymerized acrylic-based glue and kept in culture medium at $37^{\circ} \mathrm{C}$ and $5 \%$ carbon dioxide for $4 \mathrm{~d}$ before transplantation.

\section{Enzyme-linked immunosorbent assay}

The basal release of GDNF from polymer-encapsulated BHK-GDNF cells was determined by ELISA (Promega, Madison, WI). Two days after encapsulation, the amount of GDNF secreted by the capsules was measured by placing each capsule into $500 \mu \mathrm{l}$ of culture medium for $60 \mathrm{~min}$. The supernatant was removed and analyzed by ELISA. The detection limit of the assay was $20 \mathrm{pg} / \mathrm{ml}$.

\section{Behavioral analysis}

Rats were placed into cylinders attached to rotometers (Rotoscan Rotometer v5.06, Omnitech, Columbus, $\mathrm{OH}$ ). They were allowed to rest for $15 \mathrm{~min}$ to adapt to the testing environment and then were injected intraperitoneally (i.p.) with $5 \mathrm{mg} / \mathrm{kg}$ amphetamine sulfate (Research Biochemicals Incorporated, Natick, MA) dissolved in PBS. Measurement of rotational activity began $15 \mathrm{~min}$ after injection. The animals were tested for 90 min under minimal external stimuli. The lights were turned off, and the room was sealed from noise. The rotometer recorded the number of full clockwise and counterclockwise turns the animals made during the testing period. This test was performed 1 week before capsule implantation and 1 week after MFB axotomy. The change in rotation was calculated as the total number of turns the animal made under amphetamine stimulation 1 week after MFB axotomy minus the total number of turns the animal made 1 week before implantation under similar amphetamine stimulation. Clockwise turns (ipsilateral to the lesion) were counted as positive turns, whereas counterclockwise turns (contralateral to the lesion) were counted as negative turns. The net number of turns made during the entire $90 \mathrm{~min}$ testing period was counted and divided by 90 to calculate the change in the number of turns per minute. 


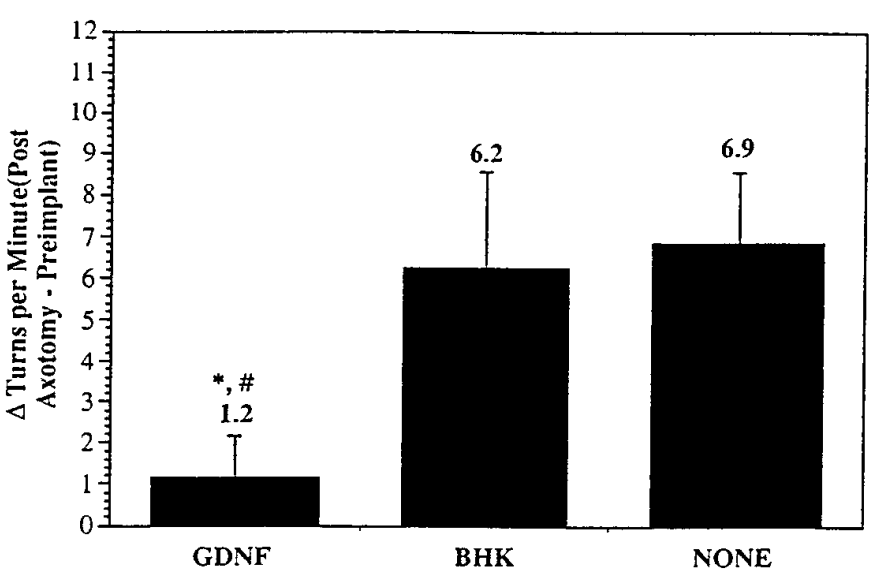

IMPLANT TYPE

Figure 2. Histogram showing the average change in turns/min experienced by the animals from 1 week before capsule implantation to 1 week after MFB axotomy induced by amphetamine stimulation $(5 \mathrm{mg} / \mathrm{kg}$, i.p. $)$. Positive values denote turns ipsilateral to the lesion. GDNF, Animals that received an implant containing BHK-GDNF cells $(n=13) ; B H K$, animals that received an implant containing BHK control cells $(n=8)$; NONE, animals that received no implant $(n=10) .{ }^{*} p<0.05$ (vs BHK), \#p $<0.02$ (vs NONE), ANOVA. Values are mean \pm SEM.

\section{Capsule implantation}

Four days after encapsulation, the animals were implanted with capsules containing either BHK-GDNF cells (GDNF, $n=13$ ) or BHK control cells (BHK, $n=8$ ). The animals were anesthetized with sodium pentobarbital $(50 \mathrm{mg} / \mathrm{kg}$, i.p.) and placed into a stereotaxic frame (David Kopf Instruments, Tujunga, CA) with the mouthbar set at $-3.3 \mathrm{~mm}$ (flat skull). A midline incision was made on the skin covering the skull, and bregma was determined. A dental drill was used to make a hole through the skull at a location $3.8 \mathrm{~mm}$ caudal to bregma and $3.5 \mathrm{~mm}$ lateral to the midline. A cannula containing the capsule, and attached to a specially designed inserter, was slowly lowered into the brain to a depth of $8.0 \mathrm{~mm}$ below dura over a period of $5 \mathrm{~min}$. The location of capsule implantation was lateral to the MFB and rostral to the SN (Fig. 1). Then the outer cannula was raised slowly while an inner obdurader remained in place to stabilize the capsule in the brain parenchyma. The skin was closed with 6-0 Vicryl (Ethicon GmbH KG, Norderstedt, Germany) in a running suture.

\section{Medial forebrain bundle axotomy}

One week after capsule implantation, a unilateral MFB axotomy was performed. The animals were reanesthetized with $50 \mathrm{mg} / \mathrm{kg}$ intraperitoneally of sodium pentobarbital and placed into a stereotaxic frame with the mouthbar set at $-3.3 \mathrm{~mm}$. A hole was drilled at the following coordinates: $3.8 \mathrm{~mm}$ caudal to bregma and $2.4 \mathrm{~mm}$ lateral to midline. An extruding Scouten wire knife (David Kopf Instruments) was lowered 8 $\mathrm{mm}$ below the dura. The wire blade was exposed $2 \mathrm{~mm}$ in the coronal plane with the tip facing medially. The knife assembly was raised slowly $2.5 \mathrm{~mm}$ and then lowered back to its original position (Knüsel et al., 1992). The wire blade was retracted, and the knife assembly was removed from the brain. The skin was closed with a 6-0 Vicryl running suture. Both surgical procedures were performed on the right side of the animals. Animals subjected to just the MFB axotomy and no capsule implantation were used as secondary controls (NONE, $n=10$ ).

\section{Striatal dopamine analysis}

After a 1 week survival period, the animals were tested again for amphetamine-induced rotational asymmetry. The next day, they were given an overdose of chloral hydrate and killed by transcardial perfusion of ice-cold PBS with $0.2 \%$ ascorbic acid and $5000 \mathrm{U}$ of heparin (Liquemin, Roche Pharma AG, Reinach, Switzerland). After PBS perfusion, the brains were extracted quickly, and the striatum was excised, wrapped in aluminum foil, and rapidly frozen on dry ice (GDNF, $n=11$; BHK, $n=$ 6 ; NONE, $n=8$ ). The capsule was removed from the brain for GDNF determination, in vitro bioactivity, and histological analysis. The mesencephalon was placed in $4 \%$ paraformaldehyde for later immunohisto-

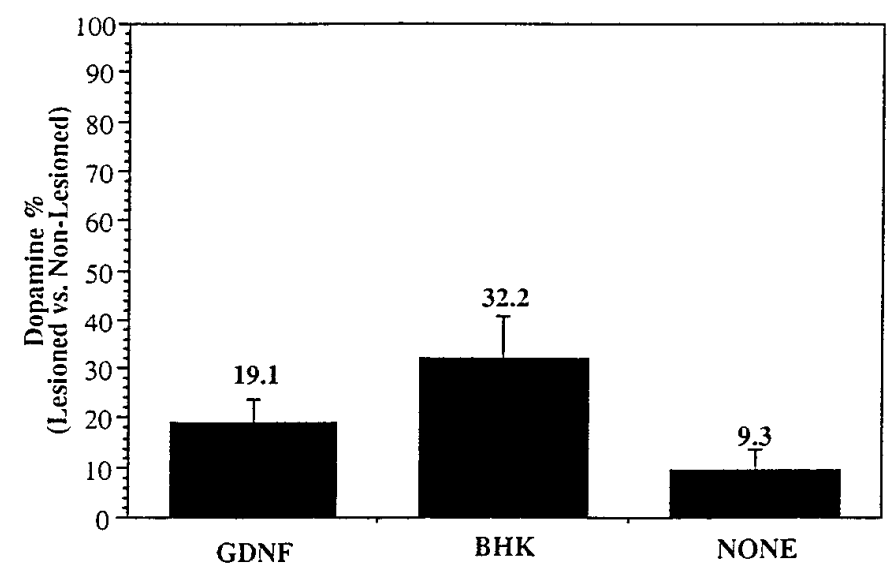

IMPLANT TYPE

Figure 3. Histogram showing the percentage of striatal dopamine content of the right (lesioned) side versus the left (nonlesioned) side, as determined by HPLC analysis. Each striatal content was normalized to the amount of protein present in the sample. GDNF, Animals that received an implant containing BHK-GDNF cells $(n=11) ; B H K$, animals that received an implant containing BHK control cells $(n=6) ; N O N E$, animals that received no implant $(n=8)$. Values are mean \pm SEM.

chemical processing. The striatum was placed into a $1.5 \mathrm{ml}$ microcentrifuge tube (Eppendorf-Netheler-Hinz-GmbH, Hamburg, Germany) containing $400 \mu \mathrm{l}$ of ice-cold $0.1 \mathrm{~N}$ perchloric acid and dissociated with a Microson Ultrasonic Cell Disruptor (Heat Systems, Farmingdale, NY). The mixture was centrifuged at $11,500 \mathrm{rpm}$ for $10 \mathrm{~min}$ at $4^{\circ} \mathrm{C}$. The supernatant was removed, placed into a filtering centrifuge tube with a 10,000 nominal molecular weight cutoff (Ultrafree-MC, Millipore, Bedford, MA), and centrifuged again. The resulting filtrate was diluted $10 \times$ with $0.1 \mathrm{~N}$ perchloric acid and analyzed on a reverse-phase $\mathrm{C} 18$ column connected to an electrochemical HPLC detector (Millipore). The mobile phase used (ClinRep, Merck AG, Basel, Switzerland) was acidified with concentrated phosphoric acid to a $\mathrm{pH}$ of 4.08 to ensure a clean separation of all catecholaminergic products. The protein content of the filtrate also was analyzed by a bicinchoninic acid (BCA) protein assay (Pierce, Rockford, IL). These values were used to normalize the amount of dopamine present in each striatal extract. The striatal dopamine content was calculated as the amount of dopamine present in the striatal extract, as determined by HPLC analysis, normalized to the amount of protein present in the extract, as determined by BCA protein analysis. All animals that had a lesioned versus nonlesioned side dopamine (DA) ratio $>50 \%$ were considered to be lesioned insufficiently and were excluded from the study.

\section{Choline acetyltransferase bioactivity}

Capsules were removed from the brains of animals and placed into cell culture medium overnight until the bioactivity of the capsule was determined. The bioactivity of GDNF being released from the capsules was assessed according to the method described by Zurn et al. (1994). Briefly, embryonic rat (E14) spinal motoneuron cultures were prepared and exposed, for $6 \mathrm{~d}$, to medium containing no trophic factor, $20 \mathrm{ng} / \mathrm{ml}$ of recombinant rat GDNF (rGDNF, PeproTech, Rocky Hill, NJ), 1 BHK control capsule, or 1 BHK-GDNF capsule. Choline acetyltransferase (ChAT) activity was determined by measuring the synthesis, in counts per minute (cpm), of $\left[{ }^{3} \mathrm{H}\right]$ acetylcholine from $\left[{ }^{3} \mathrm{H}\right]$ acetylcoenzyme A over a $20 \mathrm{~min}$ period. All values were normalized to the cpm of the control cultures that received no trophic factor.

\section{Capsule analysis}

Capsules were placed in a fixative consisting of $90 \%$ sublimated formol and $10 \%$ acetic acid for $3 \mathrm{hr}$ and then into $70 \%$ ethanol before glycol methacrylate (GMA) (Historesin, Leica, Nussloch/Heidelberg, Germany) processing. Then they were cut at 5 - $\mu \mathrm{m}$-thick sections on a Supercut 2065 (Leica), mounted on glass slides, and stained with $0.5 \%$ cresyl violet.

\section{Substantia nigra and striatum morphological analysis}

The brains were allowed to post-fix in $4 \%$ paraformaldehyde for $24-48 \mathrm{hr}$ and were transferred into $25 \%$ sucrose for $48 \mathrm{hr}$. The brains were frozen 

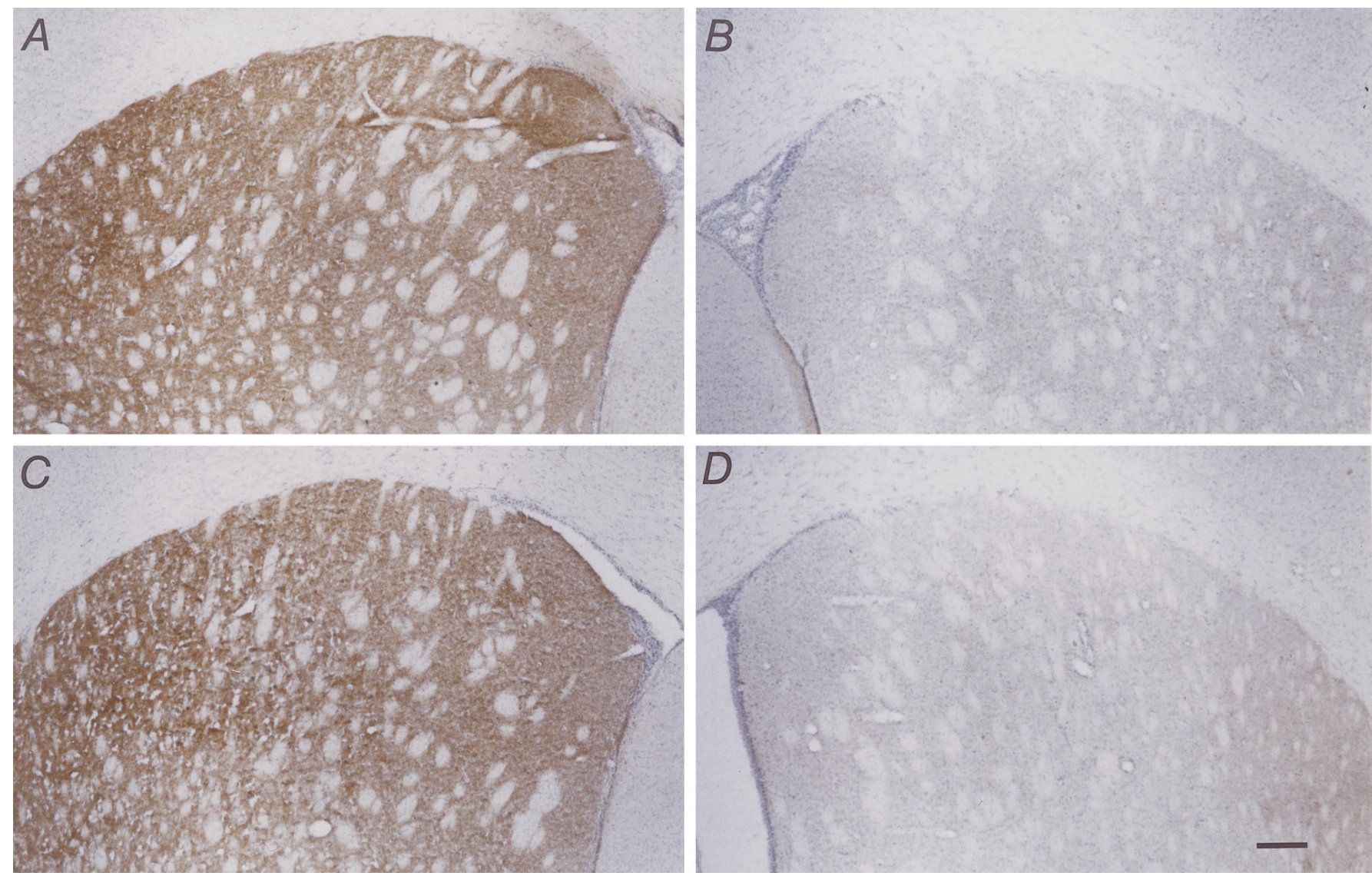

Figure 4. Micrographs showing TH-IR staining in the striatum 1 week after MFB axotomy in animals that received capsules containing either BHK-GDNF cells $(A, B)$ or BHK control cells $(C, D)$. $A$ and $C$ show the control (nonlesioned) side, whereas $B$ and $D$ show the lesioned side. Note the lack of TH-IR staining on the lesioned side in both cases, illustrating the absence of regrowth or sprouting of remaining fibers into the deinnervated striatum in either case. Scale bar, $200 \mu \mathrm{m}$.

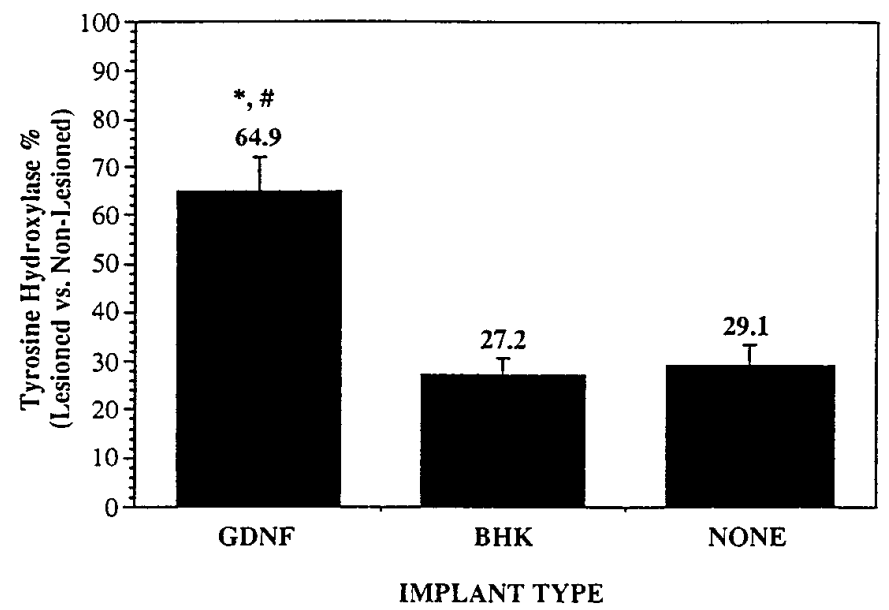

Figure 5. Histogram showing the percentage of TH-positive SN pars compacta neurons on the right (lesioned) side versus left (nonlesioned) side. Counts are based on at least 12 sections throughout the whole SN. Total number of calculations is described in Materials and Methods. ${ }^{*} p<0.0015$ (vs NONE); \#p $<0.0003$ (vs BHK), ANOVA. Values are mean \pm SEM.

in dry ice powder, and coronal sections were cut on a sliding microtome cryostat (Cryocut 1800 , Leica) at a temperature of $-24^{\circ} \mathrm{C}$ and a thickness of $25 \mu \mathrm{m}$. All slices throughout the SN were collected and stored in 48 well trays (Costar, Cambridge, MA) as free-floating sections in PBS containing $0.12 \mathrm{~mm}$ sodium azide. The trays were stored at $4^{\circ} \mathrm{C}$ until immunohistochemical processing. In six brains (2 from each group) that were not subjected to striatal dopamine analysis, the striatum was cut and every fourth section was collected in a manner similar to that of the SN.

\section{Tyrosine hydroxylase staining}

Substantia nigra. The sections first were quenched in a $0.1 \%$ solution of phenylhydrazine (Merck $\mathrm{AG}$ ) at $37^{\circ} \mathrm{C}$ for $1 \mathrm{hr}$ to reduce endogenous peroxidase activity. Then they were incubated in a blocking solution consisting of $7 \%$ normal goat serum (NGS, Dako Diagnostics AG, Zug, Switzerland) and $0.5 \%$ Triton X-100 (Sigma). The sections were agitated at a speed of $100 \mathrm{rpm}$ for $2 \mathrm{hr}$ at ambient room temperature. They were incubated in a solution containing 5\% monoclonal TH antibody (Boehringer Mannheim GmbH, Mannheim, Germany) and 5\% NGS. The sections were left overnight at $4^{\circ} \mathrm{C}$. After thorough rinsing with PBS, the sections were incubated in a $3 \%$ NGS solution containing $1 \%$ goat- $\alpha$ mouse $(\mathrm{G} \alpha \mathrm{M})$ peroxidase-antiperoxidase (PAP)-conjugated immunoglobulins (Dako Diagnostics AG) for $3 \mathrm{hr}$ at room temperature. Then the sections were visualized with a $0.5 \%$ 3,3'-diaminobenzidine (DAB, Sigma) and $0.01 \%$ hydrogen peroxide solution for $\sim 30 \mathrm{sec}$, mounted onto glass slides, and counterstained with $0.5 \%$ cresyl violet.

Striatum. The protocol was modified slightly for striatal sections. After identical phenylhydrazine quenching, the sections were agitated overnight at $100 \mathrm{rpm}$ at ambient room temperature in a blocking solution consisting of $10 \%$ NGS and $0.1 \%$ Triton X-100. This procedure was followed by a two-night incubation at $4{ }^{\circ} \mathrm{C}$ in a primary antibody solution containing $2 \%$ monoclonal TH antibody, $5 \%$ NGS, and $0.1 \%$ Triton $\mathrm{X}-100$. After thorough rinsing with PBS, the sections were agitated at 100 $\mathrm{rpm}$ and $4^{\circ} \mathrm{C}$ in a secondary antibody solution of $0.5 \% \mathrm{G} \alpha \mathrm{M}$ antibody (Vector Laboratories, Burlingame, CA), $1 \%$ NGS, $0.1 \%$ Triton X-100, and 1\% normal rat serum (NRS) (Dako Diagnostics AG) for $3 \mathrm{hr}$. The sections were, once again, rinsed with PBS and then subjected to an avidin-biotin complex reaction (Vectastain Elite ABC kit, Vector Laboratories) for $3 \mathrm{hr}$ at $4^{\circ} \mathrm{C}$ and $100 \mathrm{rpm}$. Then, after a final series of washes, 

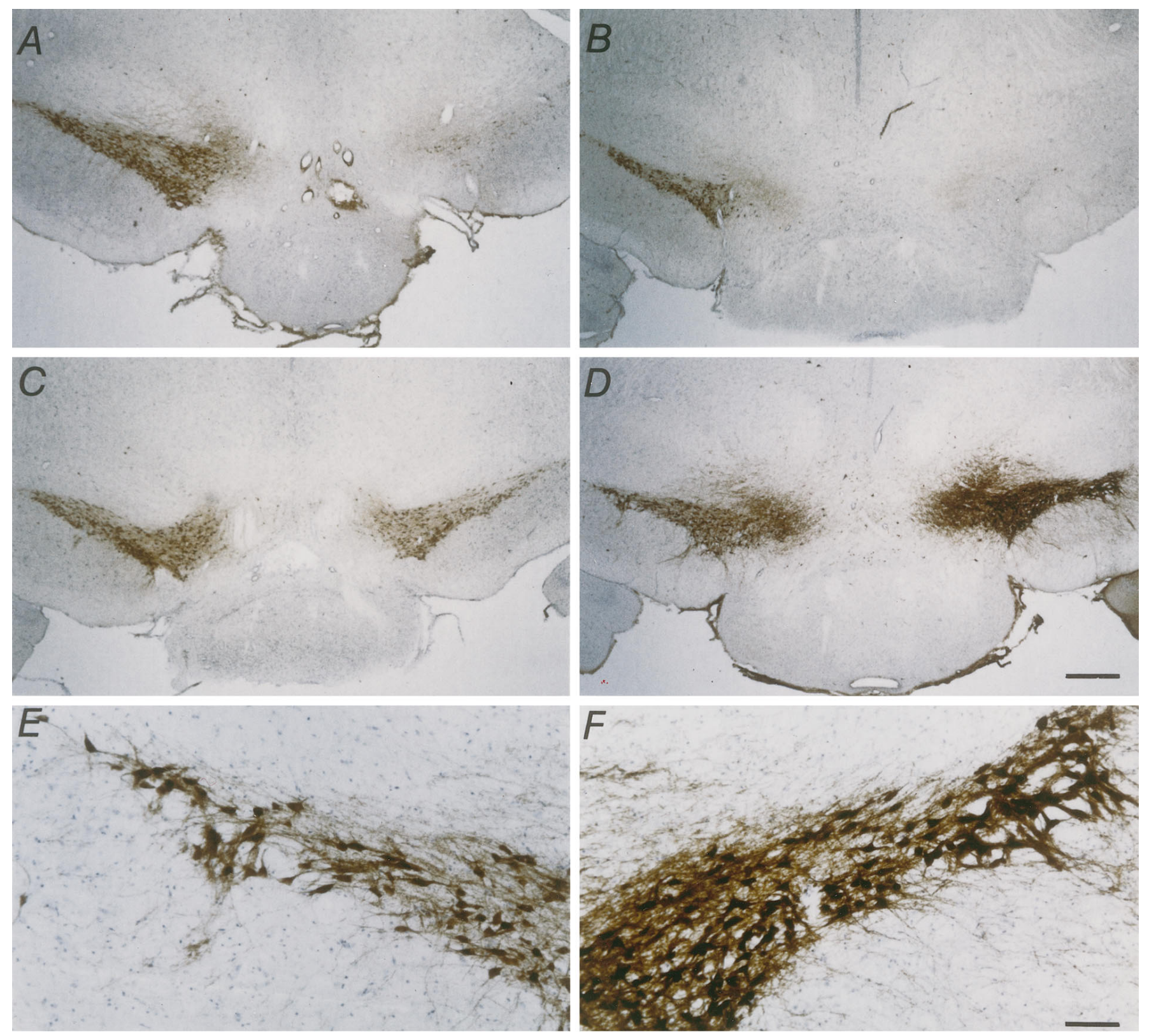

Figure 6. Micrographs showing TH-IR staining in the SN pars compacta 1 week after MFB axotomy. Low power pictures of coronal sections show the SN pars compacta. $A$, A typical control animal with no implant. $B$, A typical control animal that had received a capsule containing control BHK cells. $C$, $D$, Two typical animals that had received a capsule containing GDNF-secreting BHK cells. Note the almost total loss of TH-positive staining in the two control groups $(A, B)$, whereas the group that received BHK-GDNF cells showed substantial rescue $(C, D)$. Scale bar, $500 \mu \mathrm{m}$. High power photographs show TH-IR staining in the nonlesioned $(E)$ and lesioned $(F)$ SN pars compacta of the animal shown in $D$ that had received a BHK-GDNF implant. Note the intense TH-IR staining and extensive dendritic sprouting in the lesioned SN pars compacta $(F)$, as compared with the nonlesioned SN pars compacta (E). Scale bar, $100 \mu \mathrm{m}$.

the sections were visualized by diaminobenzidine (DAB) in a manner similar to that of the SN sections.

\section{Morphological quantification analysis}

The number of TH-IR SN pars compacta neurons was determined by counting each stained section at a magnification of $100 \times$. At least 12 sections per brain, distributed along the rostal-caudal length of the SN pars compacta, were counted. All TH-IR bodies in the A9 SN region were counted as described by Björklund and Lindvall (1984). After counting, the total number of TH-IR neurons was calculated by taking the average cell number between two counted sections and multiplying it by the number of uncounted intervening sections. So that the accuracy of this estimation method could be tested, one test brain had 48 consecutive sections stained for TH-IR. The total number was compared with a calculated number based on eight sections taken at six section intervals. The calculated number was found to be within $5 \%$ of the actual number (data not shown). This method is a specific case of the method described previously by Abercrombie (1946), in that the average neuronal diameter is very similar to the thickness of the section. The total number of TH-IR $\mathrm{SN}$ pars compacta neurons on the lesioned (right) side was divided by the total number of TH-IR SN pars compacta neurons on the nonlesioned (left) side to obtain a survival percentage.

\section{Statistical analysis}

The data obtained were analyzed for significance with ANOVA, followed by a Fisher's PLSD post hoc test (StatView 4.0, Abacus Concepts, Berkeley, CA). 

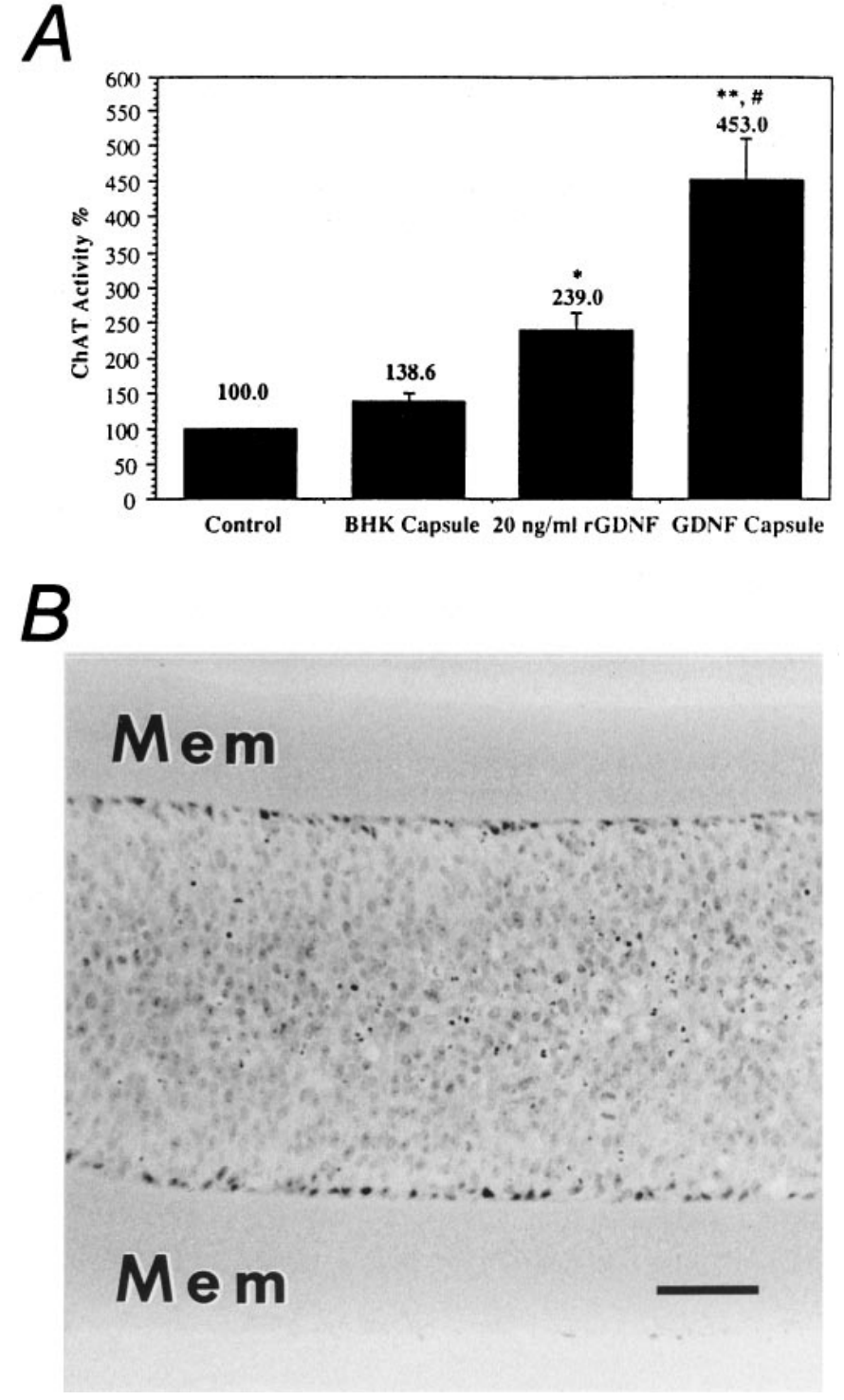

Figure 7. A, Histogram showing the effect of explanted encapsulated cells on the activity of ChAT on spinal motoneuron cultures. Control wells (negative control) received no trophic factor $(100 \%)$. Two wells per series received $20 \mathrm{ng} / \mathrm{ml}$ rat recombinant GDNF (positive control). All counts were normalized by the number of counts in the negative control. The capsules containing BHK-GDNF cells significantly increased the ChAT activity of the cultures. ${ }^{*} p<0.03$ (vs control); ${ }^{* *} p<0.0001$ (vs control); $\# p<0.0002$ (vs BHK), ANOVA. Values are mean \pm SEM. $B$, Micrograph showing encapsulated BHK-GDNF cells on explantation at 2 weeks. Note the good viability of the transplanted cells. Mem, Membrane. Scale bar, $100 \mu \mathrm{m}$.

\section{RESULTS}

\section{Behavioral analysis}

The capsules were well tolerated by all the animals; no spontaneous behavioral deficits were observed in any of the three groups. GDNF, however, significantly reduced the amphetamine-induced number of turns experienced by animals 1 week after MFB axotomy (1.2 \pm 1.0 turns/min), as compared with control animals that received either implants containing BHK cells $(6.2 \pm 2.3$ turns/ min; $p<0.03)$ or no implants $(6.9 \pm 1.7$ turns $/$ min; $p<0.02)$ (Fig. $2)$. GDNF decreased the change in the number of turns per minute to $\sim 18 \%$ of the change in the number of turns per minute of the control groups.
In 5 of the 13 animals that received BHK-GDNF implants, turning contralateral to the lesion while under the influence of amphetamine was exhibited 1 week after MFB axotomy. This behavior was not observed in any of the animals receiving control (BHK or NONE) implants.

\section{Biochemical and immunohistochemical analysis of the striatum}

The adequacy of the MFB lesion was ascertained by a decrease of striatal dopamine content. Animals that received an implant of BHK-GDNF had an average normalized striatal DA content of $19.1 \pm 4.7 \%$ (GDNF, $n=11$ ) of the lesioned side, as compared with the nonlesioned side. The lesioned versus nonlesioned DA striatal ratio was $32.2 \pm 8.7 \%$ in animals that received parent BHK-containing capsules (BHK, $n=6)$ and $9.3 \pm 4.7 \%$ in the animals lesioned only (NONE, $n=8$; Fig. 3 ).

Immunohistochemical analysis was performed on the striata of animals who were not subjected to striatal dopamine analysis (GDNF, Fig. 4A,B; BHK, Fig. 4C,D). The striata were stained for TH-IR. The nonlesioned striata showed dark, robust TH-IR staining (Fig. 4A,C), whereas the striata ipsilateral to the MFB axotomy revealed no TH-IR staining (Fig. 4B,D), showing the absence of sprouting or regeneration into the lesioned striatum.

\section{Immunohistochemical analysis of the substantia nigra}

A significant sparing of the TH-IR of the SN neurons was observed in the animals implanted with GDNF-releasing capsules, as compared with animals implanted with encapsulated BHK cells or animals that were lesioned only. BHK-GDNF-implanted animals had $64.9 \pm 7.2 \%$ TH-positive cells on the lesioned side, as compared with the nonlesioned side, whereas control animals had $27.2 \pm 3.5 \%$ (BHK; $p<0.0005)$ and $29.1 \pm 4.6 \%$ (NONE; $p<$ 0.0015) TH-positive cells (Figs. 5, 6). Similar results were obtained when the ventral tegmental area (VTA or A10) TH-positive cells also were counted and included in the calculations (data not shown). In animals that received an implant containing BHKGDNF cells, the dendritic network surrounding the cells was mostly preserved. However, in animals that received either control or no implants, the surrounding dendritic network was practically nonexistent. In one animal that received a BHK-GDNF implant (Table 1; Fig. $6 D-F$ ), the number of TH-IR neurons on the lesioned side was actually greater than the number on the control side. In this animal, an important dendritic sprouting of TH-positive fibers was observed within the nigra. However, no statistically significant correlation could be made between the number of nigral TH-IR neurons remaining and the extent of turning in animals that exhibited contralateral amphetamineinduced turning after axotomy. The same was true when comparing striatal DA content and the extent of turning (data not shown).

\section{Bioactivity and morphological appearance of the retrieved implants}

At the time of implant, capsules containing BHK-GDNF cells were determined to be releasing $\sim 5 \mathrm{ng}$ of GDNF/capsule per day (data not shown). On explantation, the capsules containing BHKGDNF cells continued to release bioactive GDNF, as measured by ChAT activity in spinal motoneuron cultures. GDNF continuously released from encapsulated BHK-GDNF cells significantly increased the ChAT activity by $453 \pm 58 \%$ ( $p<0.0001$ vs control; $p<0.0002 \mathrm{vs} \mathrm{BHK}$ ), whereas $20 \mathrm{ng} / \mathrm{ml}$ of powdered recombinant rat GDNF, used as a positive control, increased the spinal motoneuron culture ChAT activity by $238 \pm 26 \%(p<0.05$ vs 


\begin{tabular}{|c|c|c|c|}
\hline Striatal dopamine content $(\mathrm{pmol} / \mu \mathrm{g})$ & 5.77 & 1.07 & 18.54 \\
\hline Number of TH-IR neurons & 5818 & 7490 & 128.74 \\
\hline Amphetamine-induced rotations (turns/min) & $\begin{array}{l}\text { Before capsule implantation } \\
-0.33\end{array}$ & $\begin{array}{l}\text { After MFG axotomy } \\
-6.21\end{array}$ & $\begin{array}{l}\Delta \text { Turns/min } \\
-5.88\end{array}$ \\
\hline
\end{tabular}

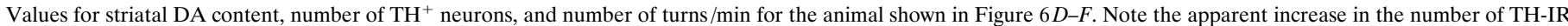

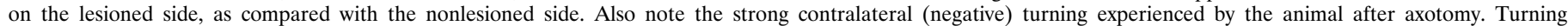
contralateral to the lesion was also seen in four additional animals that had received a capsule containing GDNF-secreting BHK cells.

control) (Fig. 7A). Capsules containing BHK control cells increased ChAT activity by $139 \pm 12 \%$. Wells receiving no trophic factors were used as controls $(100 \pm 2 \%)$.

After in vitro testing, the capsules were processed for morphological analysis. Figure $7 B$ shows a photomicrograph of a capsule retrieved at 2 weeks after implantation. All capsules contained living cells surrounding small necrotic cores.

\section{DISCUSSION}

MFB axotomy leads to the degeneration of dopaminergic neurons of the SN. The ability to prevent the degeneration of these neurons and maintain their phenotype holds promise for the treatment of PD. This report illustrates that continuous delivery of low levels of GDNF by polymer-encapsulated GDNF-secreting cells can prevent the degeneration and preserve the phenotype of the dopaminergic cell bodies that normally degenerate after a MFB axotomy. In lesioned animals that received control implants or no implants, the number of TH-IR neurons was decreased drastically throughout the entire SN pars compacta and VTA. The morphology of the cells rescued by GDNF was indistinguishable from the control nonlesioned side. The dendritic network surrounding the rescued cells was mostly preserved. Although GDNF rescued cell bodies and dendrites, it did not induce axonal regeneration into the striatum within the 2 week observation period. This is confirmed by the low levels of DA detected in the striatum after axotomy (Fig. 3) and by the absence of TH-IR staining in the striatum (Fig. 4). Longer term experiments are, however, needed to assess the capability of GDNF to induce regrowth of dopaminergic fibers within the lesioned striatum.

The ability of other neurotrophic factors, such as ciliary neurotrophic factor (CNTF) and brain-derived neurotrophic factor (BDNF), to prevent neuronal degeneration in this same axotomy model was tested previously. CNTF, delivered from a pump for $14 \mathrm{~d}(1.5 \mu \mathrm{g} / \mathrm{d})$ after lesioning, was shown to protect the axotomized neurons from degeneration but did not prevent the decrease in their TH-IR (Hagg and Varon, 1993), whereas BDNF had no rescue effect on these cells, despite numerous injection protocols [intracerebral ventricular injections, $0.58 \mu \mathrm{g} / 2 \mathrm{~d}$ for $18 \mathrm{~d}$; MFB or SN injections, $0.224 \mu \mathrm{g} / \mathrm{d}$ for $18 \mathrm{~d}$ (Knüsel et al., 1992); or SN injections, $1 \mu \mathrm{g} / 2 \mathrm{~d}$ for $18 \mathrm{~d}$ (Lapchak et al., 1993)] beginning at the time of the lesion. In contrast, the current experiments show that pretreating with continually released low levels of GDNF can prevent both the degeneration and the loss of TH-IR in the axotomized dopaminergic neurons.

In the present experiments, $73 \%$ of the SN neurons degenerate by 1 week postlesion (Fig. 2), whereas a slower degeneration has been reported by other groups using similar techniques (Knüsel et al., 1992; Hagg and Varon, 1993; Lapchak et al., 1993; Beck et al., 1995). This discrepancy could be attributable to the fact that the exact location of our axotomy is more caudal and thus closer to the cell bodies than most of the other axotomies reported (Hagg and Varon, 1993; Lapchak et al., 1993; Beck et al., 1995). The $27 \%$ survival we see after 1 week is, however, similar to the $23 \%$ endpoint survival reported by Brecknell et al. (1995) at 10 weeks. This implies that although the time point we have chosen is much shorter than that reported by other groups, it is still morphologically near the final endpoint of the degenerative process.

The amount of GDNF released in the current study is at least three orders of magnitude lower than the amount of GDNF that has been injected by other groups. Their values range from $10 \mu \mathrm{g}$ (Hudson et al., 1995) to $100 \mu \mathrm{g}$ (Hoffer et al., 1994; Bowenkamp et al., 1995) (single bolus injections). Other groups have used multiple injections that total from $20 \mu \mathrm{g}$ (Beck et al., 1995) to 70 $\mu \mathrm{g}$ (Sauer et al., 1995) at 2 weeks. Such repeated injections of GDNF have been shown to prevent the degeneration of dopaminergic neurons after MFB axotomy (Beck et al., 1995) and striatal 6-OHDA injections (Sauer et al., 1995). However, the large amounts used in these studies raise the possibility of unwanted side effects. Because it has been shown that GDNF also affects nondopaminergic systems, and given the wide expression of GDNF mRNA within both the embryonic (Schaar et al., 1993; Strömberg et al., 1993; Humpel et al., 1994; Springer et al., 1994) and adult nervous system (Pochon and Aebischer, unpublished observations), the large amounts injected also may induce changes to nondopaminergic systems.

Although the amount delivered in the present study is much lower than that administered by other groups, significant rescue of the axotomized neurons and, in some cases, increased TH-IR expression in the axotomized neurons still was observed. In one animal, the intensity of staining and the number of TH-IR cells on the lesioned side was even greater than that on the nonlesioned side (Table 1; Fig. 6D-F). This apparent increase is, in all likelihood, attributable to an upregulation of $\mathrm{TH}$ expression in dopaminergic neurons. This animal also exhibited intense amphetamine-induced turning contralateral to the lesion, as if the striatum on the lesioned side were intact and the striatum on the opposite side were lesioned. Our HPLC results have shown that this was not the case. This overcorrection of the amphetamineinduced rotation after BHK-GDNF capsule implantation was observed in 5 of 13 animals. This behavior was not observed in any animals receiving control (BHK or NONE) implants. On average, the number of turns/minute in animals that had received GDNF implants was significantly lower than that exhibited by the animals in the control groups (Fig. 5). Similar corrections were observed by Hoffer et al. (1994) and Bowenkamp et al. (1995) in 6-OHDAlesioned animals under the influence of apomorphine. Recently, Gash et al. (1996) have reported behavioral improvement in 

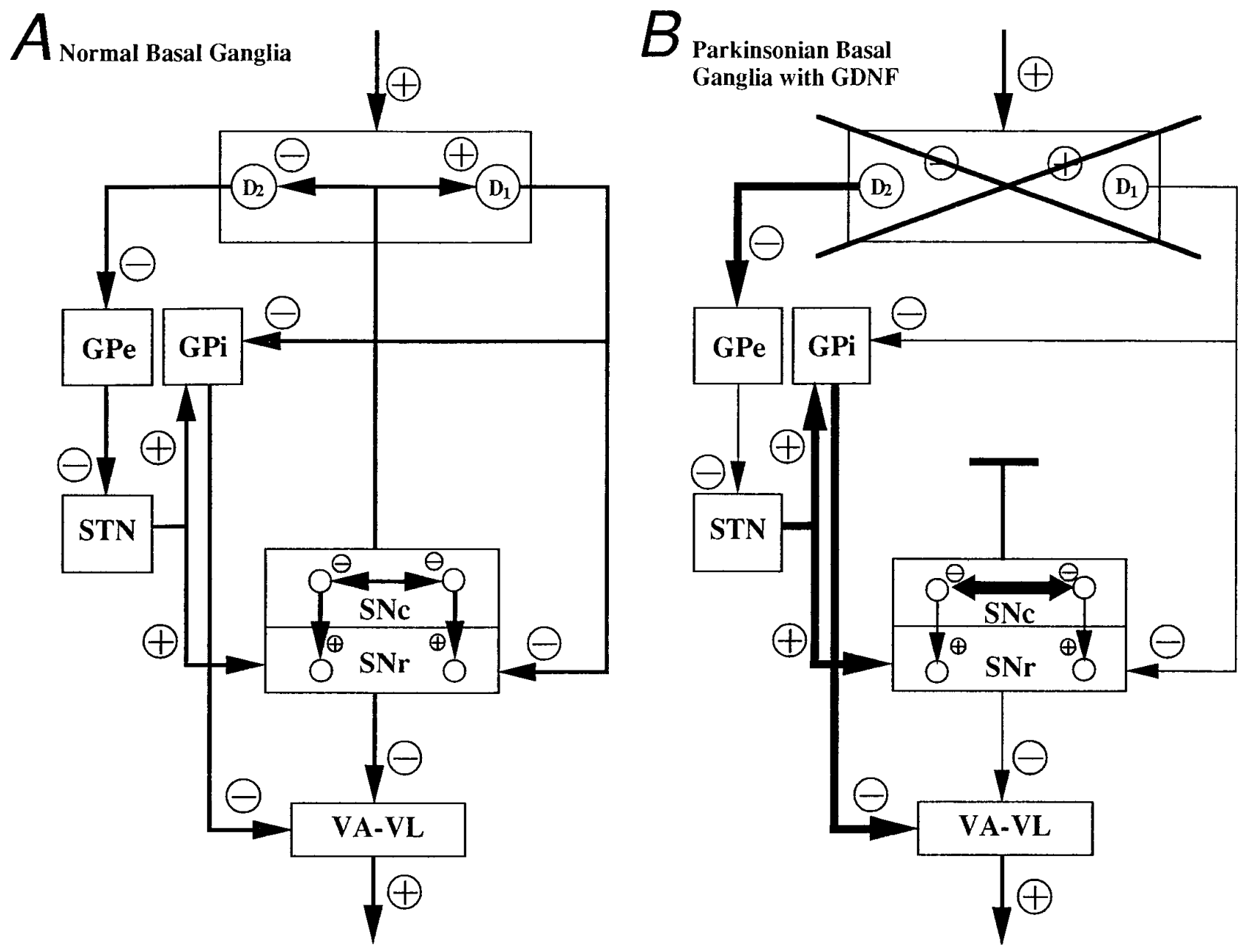

Figure 8. Diagram depicting hypothesized basal ganglia circuitry in $(A)$ normal basal ganglia. $B$, Parkinsonian basal ganglia with GDNF. GPe, Globus pallidus externus; $S T N$, subthalamic nucleus; $G P i$, globus pallidus internus (enteropeduncular nucleus); $S N c$, substantia nigra pars compacta; $S N r$, substantia nigra pars reticulata; $V A-V L$, ventroanterior and ventrolateral nuclei of the thalamus; $D_{1}, D_{2}, \mathrm{D}_{1}$ and $\mathrm{D}_{2}$ receptors of the striatum.

MPTP-treated rhesus monkeys injected with multiple bolus (100 $\mu \mathrm{g})$ injections of GDNF in the absence of recovery of dopamine in the striatum. These results seem to imply that the striatum is not absolutely necessary for the amelioration of drug-induced rotational asymmetry. The effects of GDNF on other basal ganglia functions such as sensory neglect, paw reaching, disengaging behavior, and aphagia and adipsia (Brundin and Lindvall, 1993) need to be examined.

A possible mechanism may lie in the dendro-dendritic network interconnecting the SN pars compacta neurons and/or the compacta neurons with the SN pars reticulata neurons. Ruffieux and Schultz (1980) have shown that these connections are sensitive to DA. They found that the neurons of the SN pars reticulata are activated, whereas those of the pars compacta are depressed, on the application of DA. The nigral application of GDNF, with its protective and sprouting effects on the dopaminergic SN neurons, may induce a modification of these dendro-dendritic control loops. In animals that have undergone MFB axotomy, DA stores in the preserved SN pars compacta are released at the dendritic sites, as opposed to the axonal sites in the striatum, on the application of amphetamine. This increase in DA release within the nigra may cause significant alterations in the output of the SN pars reticulata neurons (Fig. 8). This hypothesis is supported by the findings that the unilateral application of dopamine (Jackson and Kelly, 1983) or apomorphine (Kozlowski et al., 1980; LaHoste and Marshall, 1990) directly into the substantia nigra induces strong contralateral rotation in animals that had been rendered supersensitive by unilateral 6-OHDA lesioning. This indicates that supersensitivity is occurring in the SN pars compacta and not the SN pars reticulata, because supersensitivity of the SN pars reticulata receptors would activate those neurons and, consequently, further inhibit output from the thalamus. On the contrary, the development of supersensitivity in the SN pars compacta should result in the hyperactivity of the ipsilateral thalamic nuclei (i.e., strong contralateral rotations). So that this hypothesis can be examined further, direct nigral application of $\mathrm{D}_{1}$ and $\mathrm{D}_{2}$ receptor agonists into normal, lesioned, and GDNF-treated normal and lesioned SN pars compacta and SN pars reticulata is required. Recording the firing rate of pars reticulata nigral neurons in animals exhibiting overcorrection under amphetamine stimulation also should provide better insight into the functional activity of the nigral system.

In summary, the present study suggests a possible fast basal ganglia plasticity in response to certain pharmacological stimuli. This plasticity may point toward the actual mechanisms involved in the control of pharmacologically induced rotational behavior. Furthermore, this work also illustrates that continuous low level delivery of GDNF has potent dopaminergic effects in vivo, an 
observation holding promise for the treatment of Parkinson's disease.

\section{REFERENCES}

Abercrombie M (1946) Estimation of nuclear population from microtome sections. Anat Rec 94:239-247.

Aebischer P, Tresco PA, Winn SR, Greene LA, Jaeger CB (1991) Longterm cross-species brain transplantation of a polymer-encapsulated dopamine-secreting cell line. Exp Neurol 111:269-275.

Aebischer P, Schluep M, Déglon N, Joseph JM, Hirt L, Heyd B, Goddard M, Hammang JP, Zurn AD, Kato AC, Regli F, Baetge EE (1996) Intrathecal delivery of $\mathrm{CNTF}$ using encapsulated genetically modified xenogenic cells in amyotrophic lateral sclerosis patients. Nat Med 2:696-699.

Baetge EE, Suh YH, Joh TH (1986) Complete nucleotide and deduced amino acid sequence of bovine phenylethanolamine $N$-methyltransferase: partial amino acid homology with rat tyrosine hydroxylase. Proc Natl Acad Sci USA 83:5454-5458.

Beck KD, Valverde J, Alexi T, Poulsen K, Moffet B, Vandlen RA, Rosenthal A, Hefti F (1995) Mesencephalic dopaminergic neurons protected by GDNF from axotomy-induced degeneration in the adult brain. Nature 373:339-341.

Björklund A, Lindvall O (1984) Dopamine-containing systems in the CNS. In: Handbook of chemical neuroanatomy, Vol 2, Classical transmitters in the CNS, Pt 1 (Björklund A, Hökfelt T, eds), pp 55-122. Amsterdam: Elsevier.

Bowenkamp KE, Hoffman AF, Gerhardt GA, Henry MA, Biddle PT, Hoffer BJ, Granholm AC (1995) Glial cell line-derived neurotrophic factor supports survival of injured midbrain dopaminergic neurons. J Comp Neurol 355:479-489.

Brecknell JE, Dunnett SB, Fawcett JW (1995) A quantitative study of cell death in the substantia nigra following a mechanical lesion of the medial forebrain bundle. Neuroscience 64:219-227.

Brundin P, Lindvall O (1993) Transplantation in Parkinson's disease. In: Restoration of brain function by tissue transplantation (Lindvall O, ed), pp 5-17. Berlin: Springer.

Gash DM, Zhang Z, Ovadia A, Cass WA, Yi A, Simmerman L, Russell D, Martin D, Lapchak PA, Collins F, Hoffer BJ, Gerhardt GA (1996) Functional recovery in parkinsonian monkeys treated with GDNF. Nature 380:252-255.

Goetz CG, Jankovic J, Paulson GW (1989) Update on Parkinson's disease. Patient Care 23:124-162.

Hagg T, Varon S (1993) Ciliary neurotrophic factor prevents degeneration of adult rat substantia nigra dopaminergic neurons in vivo. Proc Natl Acad Sci USA 90:6315-6319.

Henderson CE, Phillips HS, Pollock RA, Davies AM, Lemeulle C, Armanini M, Simpson LC, Moffet B, Vandlen RA, Koliatsos V, Rosenthal A (1994) GDNF: a potent survival factor for motoneurons present in peripheral nerve and muscle. Science 266:1062-1064.

Hoffer BJ, Hoffman A, Bowenkamp K, Huettl P, Hudson J, Martin D, Lin LH, Gerhardt GA (1994) Glial cell line-derived neurotrophic factor reverses toxin-induced injury to midbrain dopaminergic neurons in vivo. Neurosci Lett 182:107-111.

Hudson J, Granholm AC, Gerhardt GA, Henry MA, Hoffman A, Biddle P, Leela NS, Mackerlova L, Lile JD, Collins F, Hoffer BJ (1995) Glial cell line-derived neurotrophic factor augments midbrain dopaminergic circuits in vivo. Brain Res Bull 23:425-432.

Humpel C, Hoffer B, Strömberg I, Bektesh S, Collins F, Olson L (1994) Neurons of the hippocampal formation express glial cell line-derived neurotrophic factor messenger RNA in response to kainate-induced excitation. Neuroscience 59:791-795.

Jackson EA, Kelly PH (1983) Nigral dopaminergic mechanisms in druginduced circling. Brain Res Bull 11:605-611.

Kearns CM, Gash DM (1995) GDNF protects nigral dopamine neurons against 6-hydroxydopamine in vivo. Brain Res 672:104-111.

Knüsel B, Beck KD, Winslow JW, Rosenthal A, Burton LE, Widmar HR, Nikolics K, Hefti F (1992) Brain-derived neurotrophic factor administration protects basal forebrain cholinergic but not nigral dopaminergic neurons from degenerative changes after axotomy in the adult rat brain. J Neurosci 12:4391-4402.

Kozlowski MR, Sawyer S, Marshall JF (1980) Behavioural effects and supersensitivity following nigral dopamine receptor stimulation. Nature 287:52-54

LaHoste GJ, Marshall JF (1990) Nigral $\mathrm{D}_{1}$ and striatal $\mathrm{D}_{2}$ receptors mediate the behavioral effects of dopamine agonists. Behav Brain Res 38:233-242.

Lapchak PA, Beck KD, Araujo DM, Irwin I, Langston JW, Hefti F (1993) Chronic intranigral administration of brain-derived neurotrophic factor produces striatal dopaminergic hypofunction in unlesioned adult rats and fails to attenuate the decline of striatal dopaminergic function following medial forebrain bundle transection. Neuroscience 14:639-650.

Li L, Wu W, Lin LH, Lei M, Oppenheim RW, Houenou LJ (1995) Rescue of adult mouse motoneurons from injury-induced cell death by glial cell line-derived neurotrophic factor. Proc Natl Acad Sci USA 92:9771-9775.

Lin LH, Doherty DH, Lile JD, Bektesh S, Collins F (1993) GDNF: a glial cell line-derived neurotrophic factor for midbrain dopaminergic neurons. Science 260:1130-1132.

Paxinos G, Watson C (1986) The rat brain in stereotaxic coordinates. San Diego: Academic.

Ruffieux A, Schultz W (1980) Dopaminergic activation of reticulata neurones in the substantia nigra. Nature 285:240-241.

Sagot Y, Tan SA, Hammang JP, Aebischer PA, Kato AC (1996) GDNF slows loss of motoneurons but not axonal degeneration or premature death of pmn/pmn mice. J Neurosci 16:2335-2341.

Sauer H, Rosenblad C, Björklund A (1995) Glial cell line-derived neurotrophic factor but not TGF- $\beta 3$ prevents delayed degeneration of nigral dopaminergic neurons following striatal 6-hydroxydopamine lesion. Proc Natl Acad Sci USA 92:8935-8939.

Schaar DG, Sieber BA, Dreyfus CF, Black IB (1993) Regional and cell-specific expression of GDNF in rat brain. Exp Neurol 124:368-371.

Springer JE, Mu X, Bergmann LW, Trojanowski JQ (1994) Expression of GDNF mRNA in rat and human nervous tissue. Exp Neurol 127:167-170.

Strömberg I, Björklund L, Johansson M, Tomac A, Collins F, Olson L, Hoffer B, Humpel C (1993) Glial cell line-derived neurotrophic factor is expressed in the developing but not adult striatum and stimulates developing dopamine neurons in vivo. Exp Neurol 124:401-412.

Tomac A, Lindqvist E, Lin LH, Ögren SO, Young D, Hoffer BJ, Olson L (1995) Protection and repair of the nigrostriatal dopaminergic system by GDNF in vivo. Nature 373:335-339.

Yan Q, Matheson C, Lopez OT (1995) In vivo neurotrophic effects of GDNF on neonatal and adult facial motor neurons. Nature 373:341-344.

Zurn AD, Baetge EE, Hammang JP, Tan SA, Aebischer P (1994) Glial cell line-derived neurotrophic factor (GDNF), a new neurotrophic factor for motoneurones. NeuroReport 6:113-118. 